\title{
Joint Effects of Application Communication Pattern, Job Placement and Network Routing on Fat-Tree Systems
}

\author{
Peixin Qiao \\ Illinois Institute of Technology \\ Chicago, Illinois \\ pqiao@hawk.iit.edu
}

\author{
Xin Wang \\ Illinois Institute of Technology \\ Chicago, Illinois \\ xwang149@hawk.iit.edu
}

\author{
Xu Yang \\ Illinois Institute of Technology \\ Chicago, Illinois \\ xyang56@hawk.iit.edu
}

\author{
Yuping Fan \\ Illinois Institute of Technology \\ Chicago, Illinois \\ yfan22@hawk.iit.edu
}

\author{
Zhiling Lan \\ Illinois Institute of Technology \\ Chicago, Illinois \\ lan@iit.edu
}

\begin{abstract}
Among the high-radix and low-diameter networks, fat-tree topology is commonly used in high-performance computing (HPC) and datacenter systems. Resource and job management on HPC systems is critically important to mitigate application interference in order to achieve high system performance and utilization. Preliminary studies have shown the effect of job placement on parallel scientific applications performance in fat-tree network. In this work we explore the joint effects of job placement and network routing aware of applications communication pattern on fat-tree system. Applications can be classified into various groups according to the communication patterns. We further combine various job placement policies and routing algorithms and create six different configurations. The system performance is analyzed using communication, hops, traffic, and saturation data by performing fine-grained highfidelity discrete event-driven simulation. Initial experimentation shows that the performance of HPC applications not only is related with the communication pattern, but also relies on the job placement and network routing on fat-tree systems.
\end{abstract}

\section{CCS CONCEPTS}

- Networks $\rightarrow$ Network simulations; Network performance analysis;

\section{KEYWORDS}

HPC parallel application; resource and job management; fat-tree topology; discrete event-driven simulation; interference

\section{ACM Reference Format:}

Peixin Qiao, Xin Wang, Xu Yang, Yuping Fan, and Zhiling Lan. 2018. Joint Effects of Application Communication Pattern, Job Placement and Network Routing on Fat-Tree Systems. In Proceedings of 47th International Conference

Permission to make digital or hard copies of all or part of this work for personal or classroom use is granted without fee provided that copies are not made or distributed for profit or commercial advantage and that copies bear this notice and the full citation on the first page. Copyrights for components of this work owned by others than ACM must be honored. Abstracting with credit is permitted. To copy otherwise, or republish, to post on servers or to redistribute to lists, requires prior specific permission and/or a fee. Request permissions from permissions@acm.org.

ICPP '18 Comp, August 13-16, 2018, Eugene, OR, USA

(C) 2018 Association for Computing Machinery.

ACM ISBN 978-1-4503-6523-9/18/08 .. \$15.00

https://doi.org/10.1145/3229710.3229747 on Parallel Processing Companion (ICPP '18 Comp). ACM, Eugene, Oregon, USA, 10 pages. https://doi.org/10.1145/3229710.3229747

\section{INTRODUCTION}

In a large-scale system, network topology plays a critical role because it largely determines the cost and performance of the system. Over time, the ever-increasing need for higher bandwidth and lower latency has driven the deployment of fat-tree interconnection networks [1]. In the field of high-performance computing (HPC), the fat-tree topology becomes popular and a number of Top500 systems are built on fat-tree topologies such as Stampede at Texas Advanced Computing Center (TACC) [3], Tianhe-2 at National Supercomputer Center in Guangzhou [6], Yellowstone at National Center for Atmospheric Research (NCAR) [7] and Summit at Oak Ridge National Laboratory [14]. As fat-tree network becomes increasingly used in HPC, a fundamental problem arises: how do we effectively manage critical shared network resources among multiple application processes running on fat-tree systems? For parallel applications like MPI applications, interference among application processes (e.g., MPI ranks) for shared network resources can cause serious performance variation and lead to poor application performance and low system productivity.

Several studies are dedicated to address workload interference at various system levels. Existing approaches include eliminating job interaction by separating large-sized and small-sized jobs into different system locations [9], providing contention free routes for MPI collectives [28], and decreasing interference through job placement [16] [10] or network routing [8] on fat-tree network. Workload interference is a very complicated problem, which could be caused by various configurations across the system stack. While existing studies provide useful insights of workload interference from different aspects, few have examined the problem by jointly examining the effects of application characteristics, job placement, and network routing in a holistic way.

In this work, we present an in-depth analysis of several representative applications on a pruned fat-tree system. Unlike the existing studies using socket-based co-simulation $[8,10]$, our study is based on discrete event-driven, packet-level simulation using the Codesign of Exascale Storage System (CODES) that provides higher fidelity simulation $[4,15,22,23,25,26]$. Given that workload interference only impacts communication time, in this study we focus on communication cost. We broadly classify applications into two 
groups according to their communication patterns: nearest neighbor pattern and many-to-many pattern. We conduct packet-level network simulation by using real application traces under six different combinations of job placement and routing schemes. Our analyses not only present the distribution of message latencies, but also examine traffic saturation under different configurations. By jointly examining the effects of application communication characteristics, job placement, and network routing, we make several interesting findings:

(1) For the applications dominant with nearest-neighbor communication, the use of contiguous job placement with static routing is preferred as it makes most of messages exchange through the lower level switches of the tree, thus leading to lower communication latency.

(2) For the applications dominant with many-to-many communication, the use of random job placement with adaptive routing is preferred since contiguous job placement causes many application processes contend for the limited shared network resources, thus leading to poor communication performance.

Our in-depth study and its findings could be used by system software designers to more effectively manage network resources on fat-tree systems. For instance, the key findings are important to the research on networking and job scheduling. HPC jobs are highly repetitive and have distinct communication patterns which can be anticipated and explored to mitigate resource contentions [20] [21] An intelligent job scheduler would actively collect and analyze its workload, and dynamically adjust job allocation decisions in response to job communication patterns (e.g., nearest neighbor or many-to-many dominant communication). Similarly, adaptive routing could change their routing decisions to reflect changes in workload communication characteristics and in traffic as well.

The rest of this paper is organized as follows. In section 2, we describe related works about interference study, especially in fattree topology. In section 3, we provide fat-tree network model. In section 4, we discuss methodology used in simulation. In section 5 , we analyze the application communication pattern. Section 6 shows simulation results and performance analysis. Section 7 closes this paper with our observations and some conclusions.

\section{RELATED WORK}

Interference study is a big challenge in supercomputers systems. Melanie Kambadur et al. [11] discussed eight specific sources of difficulties to measure interference in live datacenters. They proposed a generally applicable methodology for measuring application interference in the restrictive environment of a datacenter. First, they measured the performance data in small samples using several remote collection machines. Next the interference relationships between applications were identified. Finally, they used the aggregated performance data and the baseline performance indicators to analyze system-wide application interference. In addition, the evaluation of the presented techniques was implemented in live production datacenter workloads. Christina et al. [5] further quantified interference in resources that span the CPU, cache hierarchy, memory, storage and networking subsystems. They presented a novel workload suite, iBench that helps measure interference for datacenters application in quantity.

To mitigate interference and achieve high utilization, resource and job management is significantly important for supercomputer systems. Ana Jokanovic et al. [9] presented a novel technique for mitigating interference by reducing job interactions in their neighborhoods, which is called quiet neighborhoods. This resource manager creates two phases. In first phase, it creates multiple virtual blocks in system and one side is for big size jobs, and the other side is for small size jobs. In second phase, each coming job is assigned to the specific block according to their job size. They evaluated their resource manager using a workload trace obtained from the MareNostrum system. In addition, their technique further has potential hardware and power savings.

Javier Navaridas et al. [16] further investigated the resource management deeply. They studied the influence of job and task placement on the performance of application. They analyzed their resource manager in the torus and fat-tree topologies. They performed trace-driven simulation and studied the influence of communication locality and overhead on the performance of application. However, they overlook the influence of application communication pattern on interference and performance. Xu Yang, Zhou Zhou et al. [27, 30-32] studied resource and job management on torus and $5 \mathrm{D}$ torus networks in various aspects such as locality-aware scheduling and relaxing allocation constraints.

These related works provide interference measure strategy, mitigate method and various resource and job management. However, to the best of our knowledge, there is no research studying the joint influences of application communication pattern, job placement and network routing on fat-tree systems in event-driven, packetlevel simulations. In our paper, we perform our simulation using real application traces and select applications with representative communication pattern. We use fine-grained high-fidelity simulator, CODES and these selected applications can be implemented through event-driven, packet-level simulations on CODES. We combine job placement policies and routing algorithms and have six different configurations. We further analyze how these resource management configurations affect the system performance using the communication, traffic, hop and saturation data.

\section{FAT-TREE NETWORK}

Here we describe the commonly used fat-tree models [1, 17]. In particular, we discuss pruned fat-tree configuration, which is widely deployed in production systems.

\subsection{Two- or Three-Tier Network Topology}

Typical data center network topology consists of either two- or three-level of switches or routers. For example, the Stampede system at TACC uses two-tier fat-tree model with an oversubscription of 5:4, which means $80 \%$ of available host bandwidth is available [3]. Specifically, on Stampede, one 36-port switch is connected with 20 nodes and 16 ports of other switches. The MareNostrum system at Barcelona Supercomputing Center uses three-tier fat-tree network with an oversubscription of 1:1 [9]. This network is full bisection bandwidth interconnection, which means one half of the nodes can communicate simultaneously with the other half of the nodes. 


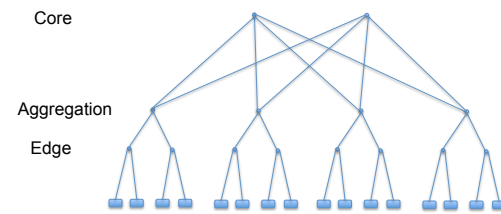

(a) Three-Tier Network Topology

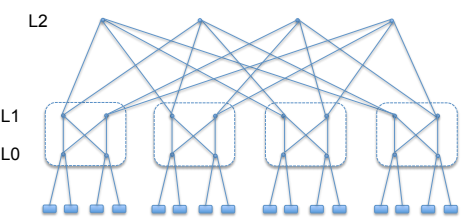

(b) k-ary Fat-Tree Architecture

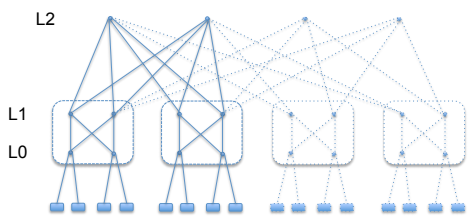

(c) Pruned Fat-Tree configuration

Figure 1: Fat-Tree Network Model

An example of three-tier fat-tree topology is shown in Figure 1(a). There are three levels of switches (or routers). Each switch has 4 ports. It is with an oversubscription of 2:1 since the switch at the edge level is connected with two hosts and one switch port at the aggregation level. This topology has a serious shortcoming, that is, the excessive cost of switches with aggregate high port speeds in higher levels of the hierarchy [1].

\section{2 k-ary Fat-Tree Architecture}

To resolve the excessive cost problem in the two- or three-tier fattree model, Al-Fares et al. proposed a fat-tree network architecture, which is shown in Figure 1(b) [1]. For a given radix of $k$ (the number of ports in one switch), there are $k$ pods in the fat-tree topology. In each pod, there are $k$ switches equally distributed into two layers and each switch is with $k$ ports. Each switch at lever L0 is connected to $k / 2$ computer nodes and $k / 2$ switches at level L1 (1 port for each). Similarly, each switch at level L1 is connected to $\mathrm{k} / 2$ switches at level L0 and k/2 switches at level L2. Hence, the fat-tree network has full bisection bandwidth. Therefore, each pod connects with $(k / 2) \cdot(k / 2)$ computer nodes and $k \cdot(k / 2) \cdot(k / 2)=k^{3} / 4$ computer nodes are interconnected in the system. Figure 1 shows a 4 -ary fat-tree, which means radix is 4 , so there are 4 computer nodes in each pod and the system contains 16 computer nodes in total.

\subsection{Pruned Fat-Tree Configuration}

For a real system with specific number of nodes, the full bisection fat-tree topology needs to be modified. Thus, pruned fat-tree configuration is commonly adopted in production systems such as Cab at Lawrence Livermore National Laboratory [13] and Summit at Oak Ridge National Laboratory [14]. Based on the k-ary fat-tree network model, some pods are pruned to match the real system. For example, a pruned fat-tree model with two pods is illustrated in Figure 1(c).

In this study, we investigate a 648-node pruned fat-tree system, which is denoted by XGFT $(3 ; 18,18,36 ; 1,18,18)[18]$. The network is built with 36-port switches. Each switch at level L0 is connected to 18 computer nodes and there are 18 switches at level L0 and 18 switches at level L1 in one pod, so there are 324 computer nodes connected to the switches in one pod. In total, there are 2 pods and $36 \mathrm{~L} 0$ switches, $36 \mathrm{~L} 1$ switches and $18 \mathrm{~L} 2$ switches in our system. Each switch has 36 ports, and the network interconnection keeps full-bisection bandwidth. To scale down the system proportionally, all the switches are simulated with a 90ns traversal delay and each virtual channel with $4 \mathrm{~KB}$ buffer size per port. The bandwidth between computer nodes and switches and the bandwidth between switches are both set as $1.25 \mathrm{~GB} / \mathrm{s}$.

\section{METHODOLOGY}

In this section, we describe the applications studied in this world. We also present job placement and network routing configurations.

\subsection{Application Traces}

In this paper, we focus on two communication patterns commonly observed in HPC applications. Specifically, we select three applications: Algebraic MultiGrid Solver (AMG), Crystal Router (CrystalRouter) and Geometric Multigrid V-Cycle from Production Elliptic Solver (MultiGrid). Both AMG and CrystalRouter are dominant with nearest-neighbor communication, whereas MultiGrid is dominant with many-to-many communication. In addition, the simulations only account for communication time and don't use computation time.

AMG: The AMG is a parallel algebraic multigrid solver and preconditioner for unstructured mesh physics packages to solve large problems posed on unstructured grids in linear systems. An AMG solver for massively parallel computers was developed at Lawrence Livermore National Laboratory (LLNL) [24]. The DUMPI MPI trace collected the overall communications data and mesh topology data in AMG code.

CrystalRouter: Crystal Router is a trace of an extracted communication kernel for the Nek5000 production application, which is an open source, highly scalable and portable spectral element code[19]. It was developed by Argonne National Laboratory (ANL). The Crystal Router communication pattern only requires the amount of memory needed to transfer the data.

MultiGrid: Geometric Multigrid V-Cycle from production elliptic solver BoxLib is a software library containing all the functionality to write massively parallel, block-structured adaptive mesh refinement (AMR) applications in two and three dimensions using structured grid physics packages. It was developed by Lawrence Berkeley National Laboratory (LBNL) [2].

\subsection{Job Placement Policies}

Job placement is a process of mapping application processes to available computer nodes. In this paper, we investigate two placement policies from the previous work [25] and also present a new job placement policy named group placement.

(1) Contiguous: Each job is assigned of a consecutive set of available nodes. In this policy, all ports in a switch is first fully 
used and then next switch in the same pod will be available. If extra resources are still required, computer nodes connected to switches in next pod will be assigned as the same rule. This method is with good spacial locality, which can reduce the number of hops, however, it tend to increase congestion in traffic links of one switch or one pod.

(2) Random: In this policy, a job receives a random selection of available computer nodes in the system. In the fat-tree topology, it allocates all the computer nodes randomly rather than consider which pod and which switch is priority choice. This policy breaks the spacial locality of the communication and may increase the number of hops as packets traverse through switches to their destination, while it probably decrease the congestion in traffic links of one switch or one pod.

(3) Group: We combine the computer nodes connected to one level L0 switch as a group, which means 18 nodes in one group on our system configuration. In this policy, one switch in level L0 is randomly chosen and all the available computer nodes connected to this switch are first assigned to the job. If the job requires more computer nodes, it will gets another level L0 switch randomly. This policy balances the spacial locality and randomness and aims to achieve better performance.

\subsection{Network Routing}

Routing determines the physical path traversed by packets from the source switch to the destination switch in a network. On fat-tree systems, two commonly used routing algorithms are:

(1) Static: One of the state-of-the-art routing algorithms is presented to provide a congestion-free, all-to-all shift pattern leveraging on the InfiniBand static routing capability [29]. Each destination is assigned a local identifier (LID) and each switch has a linear forwarding table (LFT) containing outgoing port, through which packets are forwarded as a function of the packet destination LID. The routing engine calculates and writes out the extracted LFT for the virtual InfiniBand network, which are incorporated to accurately simulate the application traffic via deterministic static-destination routing algorithm in our fat-tree network [23].

(2) Adaptive: In this policy, the path a packet traverses is adaptively chosen to balance traffic on different links locally [23]. This algorithm utilizes token-count mechanism. Each port is assigned a initial value to store the packets being forwarded. The value will be decreased if a packet comes while it will increase if a packet leaves from the port. Among the ports, the port with highest value will be selected to enqueue the packet.

\subsection{Evaluation Strategy}

We adopt discrete event-driven, packet-level simulations for performance analysis. Specifically, we use the CODES toolkit (Co-design of Exascale Storage System). CODES provides detailed communication statistics for analyzing the performance of each application. In detail, application communication traces are collected using the DUMPI trace library [12], and then the traces can be read by CODES to extract communication data. Specially, it can keep record of communication time, computing time, message hops, send time and receive time for each MPI rank (i.e., application process), network traffic and saturation time for each port of network devices.

Intuitively, communication time is a good performance evaluation metrics. The communication time refers to the time each MPI rank takes between starting to send the message and finishing the communication. Since we use CODES simulator, the absolute communication time for each rank can be collected, the average of which is the evaluation of the performance of each application.

While, some network metrics such as hops, traffic and saturation can help us explore why these applications have different performance behaviors. Hops refer to the number of links the packets travel from the source to the destination. Data packets pass through switches when they traverse through the network in our fat-tree topology. Each time packets pass through a switch, an hop occur. CODES collects hops for each MPI rank. Average hops can evaluate the average number of links traversed for each application. The traffic refers to the amount of data going through the network devices. CODES provides us with the traffic of each port of each switch. In our work, we aggregate the traffic of all the ports of each switch. Then, we analyze the traffic of each switch. The saturation time refers to the time period when the buffer of specific port in a switch is full. We collect the saturation data to measure network congestion.

\section{APPLICATION COMMUNICATION CHARACTERISTICS}

As we mentioned above, we selected three representative applications from DOE Design Forward Project. First, we analyze the data intensity as shown in Table 1.

\section{Table 1: Application Communication Data}

\begin{tabular}{c|c|c|c}
\hline App & \#Ranks & Total Data & Avg. Data/Rank \\
\hline AMG & 216 & $130 \mathrm{MB}$ & $0.6 \mathrm{MB}$ \\
\hline CrystalRouter & 100 & $3500 \mathrm{MB}$ & $35 \mathrm{MB}$ \\
\hline MultiGrid & 125 & $625 \mathrm{MB}$ & $5 \mathrm{MB}$ \\
\hline
\end{tabular}

Figure 2 shows the application communication matrices of these applications. In these figures, the number beside the color bar is the communication size in bytes and the numbers in $x$ axis and $y$ axis are the MPI ranks. The dark red area means that there are a lot of communication data in bytes, the light red area represents that there is few message transfer, while there is no communication in the white area.

As the figure 2(a) shows, most of dark red area occurs at the diagonal area traversing from the lower left corner to the upper right corner. The color of the whole figure is light-red, which means there is few communication among all ranks. However, compared with the dark-red area, the communication data in size is negligible, which can be neglected. In addition, there is nearly no white area in the figure. Specifically, most of communication data happens among not more than 18 MPI ranks, while some MPI ranks communicate with MPI ranks for a distance of 36 MPI ranks. Here we should notice that there are 18 ports in one level L0 switch connected to 


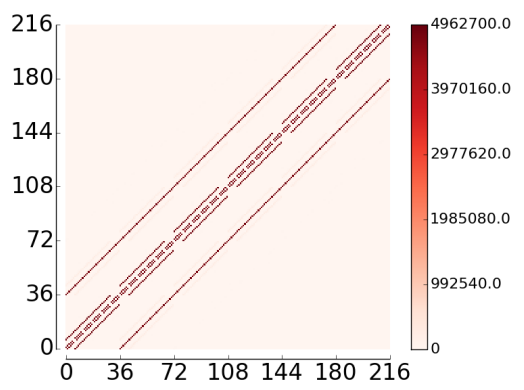

(a) AMG

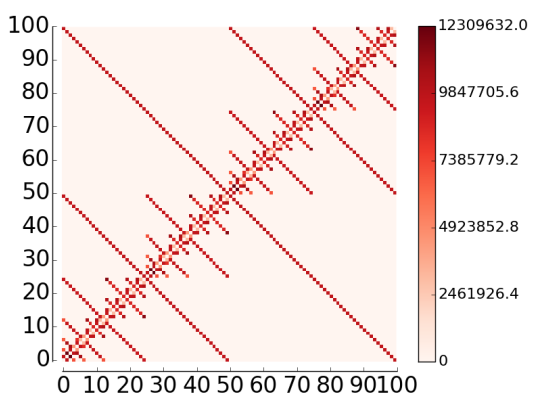

(b) CrystalRouter

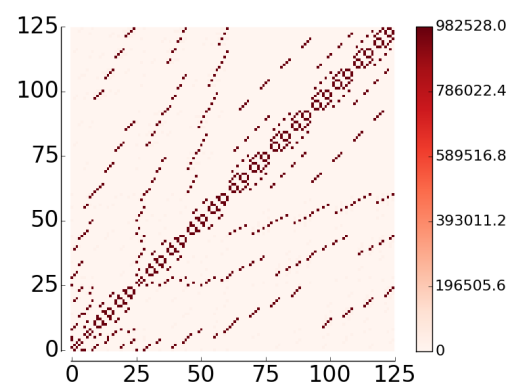

(c) MultiGrid

Figure 2: Application Communication Matrices

computer nodes, therefore, most of communication may be done under level L0 switches. Hence, for AMG application, each MPI rank tends to send the message to close MPI ranks and receives the message from close MPI ranks, which should be classified as nearest-neighbor communication pattern.

We show the communication pattern of CrystalRouter application in Figure 2(b). Intuitively, the communication pattern in this figure is different from that of not only MultiGrid application, but also AMG application. Let us analyze it quantitatively. In this figure, most of communication data occurs at the diagonal area traversing from the lower left corner to the upper right corner, while some message communicate on the perpendicular diagonal of it. However, most of packets are sent and received within 18 MPI ranks, which we should also notify here. Compared with the amount of these packets, other communication packets are negligible. Hence, we classify this communication pattern as nearest-neighbor neighbor communication pattern.

For MultiGrid application, the communication pattern is shown as Figure 2(c). The color and the numbers have the same meaning as Figure 2(a). Rather than focusing on the diagonal area, the communication data is distributed randomly on the whole area in the figure. Some MPI ranks traverses from source to destination within 18 MPI ranks, while some MPI ranks communicate with a distance of more than 18 MPI ranks. Therefore, this communication pattern of MultiGrid application is classified as many-to-many communication pattern.

After analyzing the communication pattern of AMG, MultiGrid and CrystalRouter, we classify them into two different groups. AMG and CrystalRouter are considered as nearest-neighbor communication applications, while MultiGrid is classified as a many-to-many communication application.

\section{PERFORMANCE ANALYSIS}

In this paper, we examine various combinations of job placement policies ad network routing mechanisms (see Table 2).

For each configuration, we analyze communication time for each application. We also analyze average hops, traffic amount, and saturation of each switch in different levels.
Table 2: Combinations of Job Placement and Routing Algorithms

\begin{tabular}{c|c|c}
\hline \multirow{2}{*}{ Job Placement } & \multicolumn{2}{|c}{ Routing Policies } \\
\cline { 2 - 3 } & Static & Adaptive \\
\hline Contiguous & cont-sta & cont-adp \\
\hline Group & grou-sta & grou-adp \\
\hline Random & rand-sta & rand-adp \\
\hline
\end{tabular}

\subsection{Nearest-Neighbor Communication}

The communication time shows the performance of the applications for different job placement policies and network routing configurations intuitively, since it represents the time it takes to send and receive the message and the amount of the message is constant for different configurations, which is shown in Figure 3. For each kind of configuration, CODES provides the communication time for each MPI rank. We collect all of those data and draw the figure using boxplot with matplotlib. On each box in Figure 3, the central mark is the median, the edges of the box are the lower hinge defined as the 25 th percentile and the upper hinge defined as the 75th percentile, and the little cycles outside of box mean the most extreme data points not considered, which are plotted individually.

Observation 1: For the applications dominant with nearest-neighbor communication such as AMG and CrystalRouter, contiguous job placement configuration outperforms group job placement configuration and group job placement outperforms random job placement configuration with the same routing algorithm. For CrystalRouter application, no matter which job placement is deployed, static routing policy performs better than adaptive routing policy. For AMG application, with contiguous or group job placement, static routing outperforms adaptive routing, however, with random job placement, adaptive routing outperforms static routing.

Figure 3(a) shows the communication time for AMG application. From the figure, we know that deploying the same routing, it takes more time to transfer the message for group job placement than contiguous job placement and less time than random job placement on average. With contiguous or group job placement the communication time of static routing is less than that of adaptive routing, however, with random job placement the communication time of 


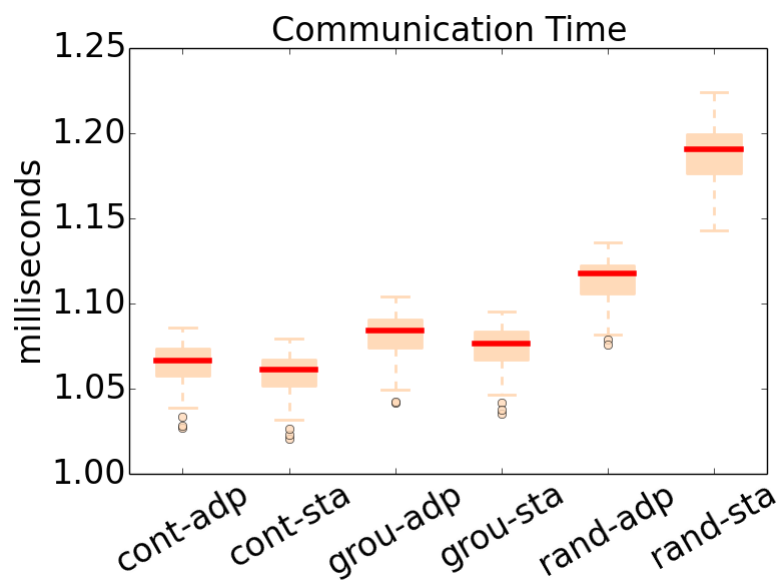

(a) AMG

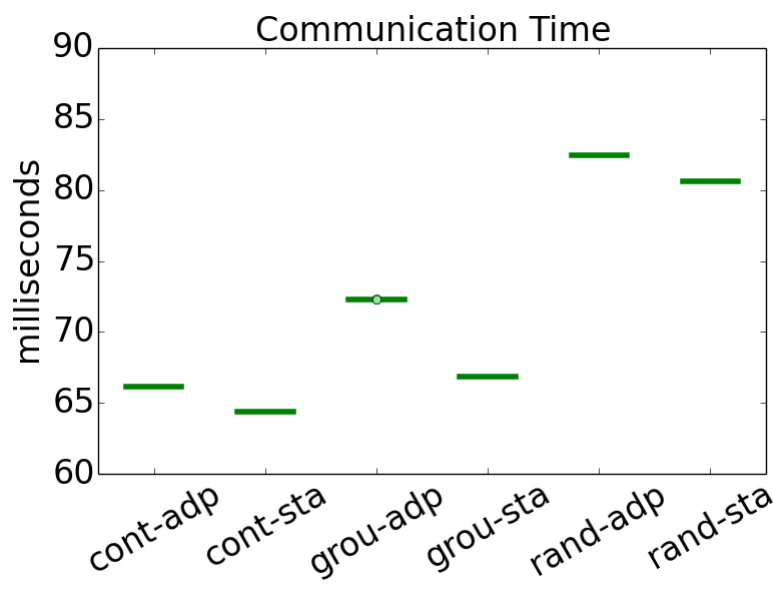

(b) CrystalRouter

Figure 3: Communication Time for AMG and CrystalRouter Applications.

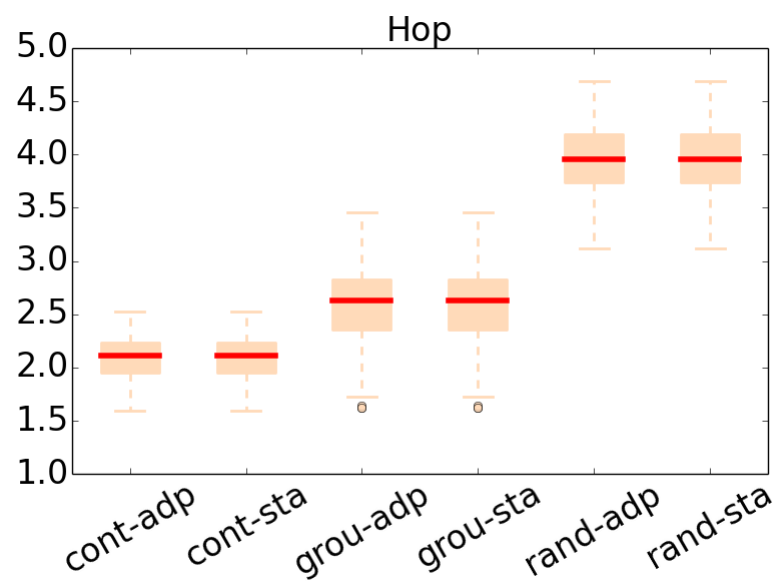

(a) AMG

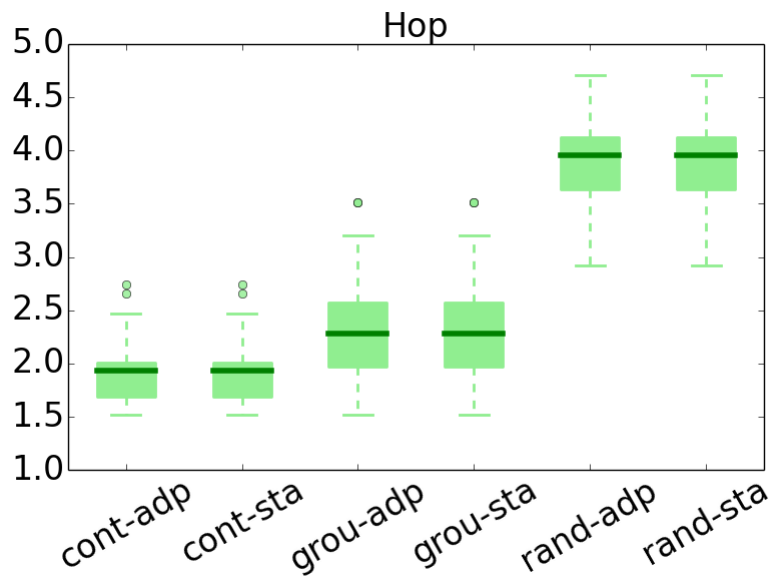

(b) CrystalRouter

Figure 4: Hops for AMG and CrystalRouter Applications.

adaptive routing is less than that of static routing. The communication time for CrystalRouter is shown in Figure 3(b). From the figure, we know that using the same routing, the communication time of contiguous job placement is less than that of group job placement and the communication time of group job placement is less than that of random job placement. The communication time of static routing is less than that of adaptive routing if using the same job placement configuration. Here we notify that the reason why CrystalRouter application communication time figure doesn't have "divergence" is that the data intensity of CrystalRouter is large. To know why the communication time for different configurations behaves like this, we have the following analysis.

Analysis: Hops describe the number of links the message traverse. Hence, the more hops, the more time it may take for the same message on communication. Figure 4 refers to the hops for different job placement policies and routing algorithms configurations. In Figure 4(a), using the same job placement, the hops of static routing algorithm and adaptive routing algorithm are nearly the same for AMG application. In addition, the hop of contiguous job placement is less than that of group job placement and the hop of group job placement is less than that of random job placement. For contiguous job placement policy, it may only send and receive the message in the lower level switches since it takes less hops for communication, however, the communication may occurs in all level switches since it takes much more hops for random job placement policy. The average hops of different configurations for CrystalRouter application is shown as Figure 4(b), in which we have the same analysis results. The hop of static routing policy is 
nearly the same as that of adaptive routing algorithm, and the hop of contiguous job placement is less than that of group job placement policy and the hops of group job placement policy is also less than those of random job placement policy.

Figure 5 shows the traffic data for AMG application. We know that for contiguous job placement, most of traffic occurs in lower level switches (Level L0 and Level L1), and there is nearly no traffic in Level L2 switches. For group job placement and random job placement, the network traffic is distributed at all level switches. Therefore, packets traverse less switches for contiguous job placement policy, which is consistent with the behavior of average hops. Recall in last section we have notified that there are 18 ports within one switch and most of messages communicate within 18 MPI ranks. Hence, for AMG applications, the network traffic occurs in lower level switches and takes few hops for contiguous job placement, which means that packets travel from source to destination with a short distance, and there is few congestion, so the communication time is less than that of group job placement and random job placement. With contiguous job placement, the traffic of static routing is distributed more balanced than adaptive routing, but from Figure 6 the saturation doesn't differ too much for static routing and adaptive routing. Hence, the network traffic and saturation cannot explain why with random job placement, adaptive routing outperforms static routing for AMG application. CODES simulator don't record startup time (start one thread, network interface, routing algorithm and so on). We will explore these aspects in detail in future work.

For CrystalRouter application, let us see the Figure 7. For contiguous job placement, there is nearly no traffic data in Level L2 switches and most of traffic occurs in lower level switches (Level L0 and Level L1). For group job placement and random job placement, the traffic is distributed at all level switches. So again, it takes few hops for contiguous job placement, which means that packets travel from source to destination with a short distance, and there is few congestion, so the communication time is less than that of group job placement and random job placement. See the Figure 8, there is nearly no saturation in Level L0 and Level L2 switches for contiguous job placement, and there is more saturation for adaptive routing than static routing. Hence, the communication time of contiguous job placement and static routing is least among these six configurations.

Conclusion: According to the analysis above, we conclude that for applications dominant with nearest-neighbor communication pattern, most of traffic of contiguous job placement configuration with static routing occurs in lower level switches on the fat-tree network since most MPI ranks can finish their communication only using one level switch or two level switches, and there is no obvious more saturation for this configuration, so the communication time is least among six different configurations in our simulation.

\subsection{Many-To-Many Communication}

In this subsection, we will analyze the performance of many-tomany communication pattern application, which is MultiGrid application. First we describe the communication time of different job placement and network routing configurations for MultiGrid application. Then we continue discussing the average hops of different configurations. We also discuss the network traffic and saturation time to explain why the communication time behaves like this for different configurations.

Observation 2: For the applications dominant with many-tomany communication such as MultiGrid, random job placement outperforms group job placement and group job placement outperforms contiguous job placement with same routing algorithm. No matter which job placement is deployed, adaptive routing policy performs better than static routing policy.

The communication of time for MultiGrid application is shown in Figure 9(a). From the figure, we know the communication time of random job placement is less than that of that group job placement and the communication time of group job placement is less than that of contiguous job placement if deploying the same routing algorithm. Figure 9(a) shows whichever job placement is used, the communication time of adaptive routing policy is less than that of static routing policy. To know why they behave like this and that as Observation 2, we provide the following analysis.

Analysis: As shown in Figure 9(b), the hops of different job placement and routing configurations for MultiGrid application behave similarly as those for AMG and CrystalRouter applications. The message for random job placement configuration traverses more switches for communication compared with group job placement configuration and the message for group job placement configuration traverses more switches compared with contiguous job placement configuration. However, MultiGrid application is with many-to-many communication pattern. The more switches the packets travel through, the communication time it needs less since it will avoid congestion, which will be discussed in detail with the network traffic and saturation data.

The Figure 10 shows the traffic for MultiGrid application. From the figure, we know that most of the traffic occurs in lower level switches for contiguous job placement configuration. Hence, it will lead to more congestion in lower level switches since MPI ranks communicate with other multiple MPI ranks in chaotic manner, which is shown in Figure 11. The saturation of contiguous job placement occurs only in lower level switches. According to the above analysis, we can know that for contiguous job placement configuration, it only uses lower level switches to communicate. Therefore, the communication time of contiguous job placement configuration is more than that of other configurations. Compared with random job placement, the saturation of group job placement also occurs more in lower level switches and the message traverses less switches. With the similar reason, the communication time of group job placement configuration is more than that of random job placement configuration. However, the average traffic amount and saturation time of one switch cannot explain why adaptive routing outperforms static routing whichever job placement is used. We will also explore the reason in some aspects mentioned above in our future work.

Conclusion: According to the analysis above, we conclude that for many-to-many communication pattern applications, most of traffic of contiguous job placement configuration occurs in lower level switches on the fat-tree network and it lead to more congestion, so random job placement configuration performs better than other job placement policies. While adaptive routing outperforms static routing policy using the same job placement. 


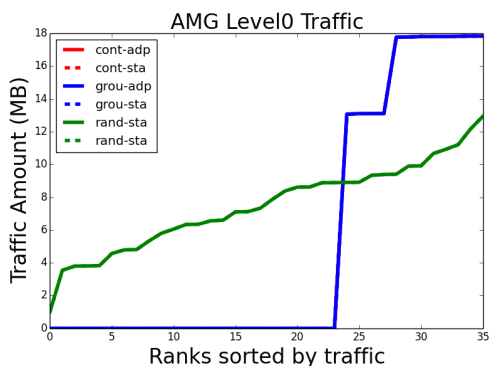

(a) Level L0

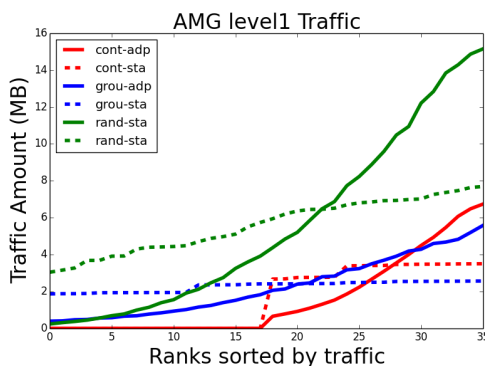

(b) Level L1

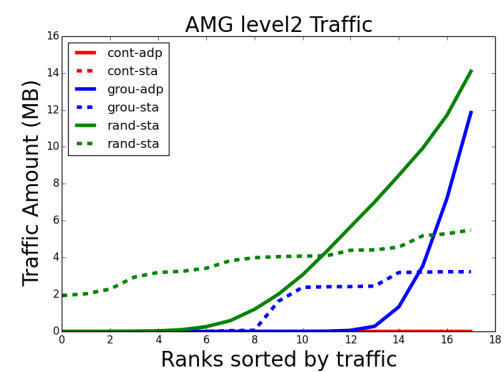

(c) Level L2

Figure 5: Traffic Amount for AMG Application.

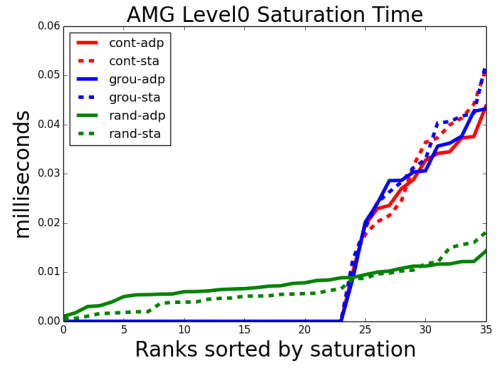

(a) Level L0

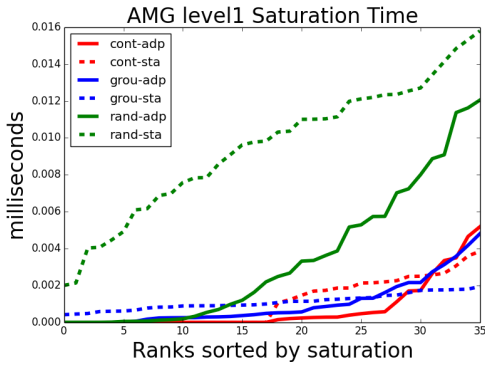

(b) Level L1

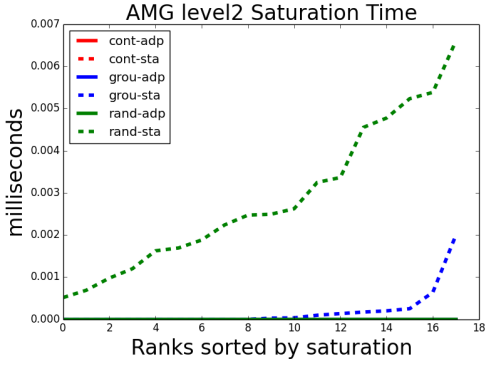

(c) Level L2

Figure 6: Saturation Time for AMG Application.

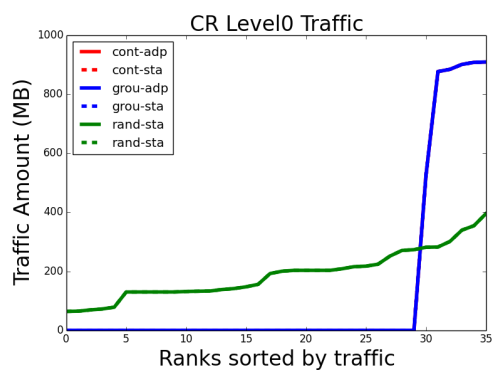

(a) Level Lo

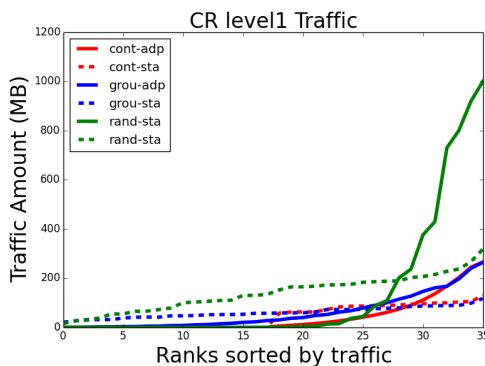

(b) Level L1

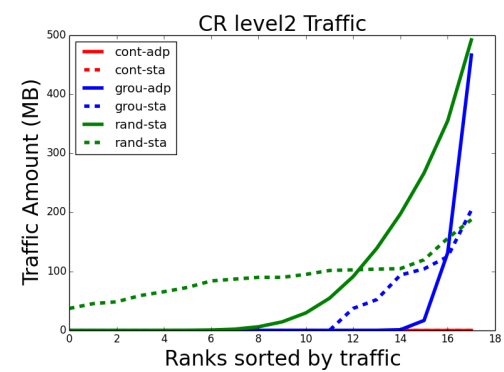

(c) Level L2

Figure 7: Traffic Amount for CrystalRouter Application.

\section{CONCLUSIONS AND FUTURE WORK}

In this work, we have presented an in-depth analysis of several representative applications on a pruned fat-tree system by means of fine-grained network simulations. We use real application traces as well as discrete event-driven simulator (CODES) to analyze resource management. We analyzed applications communication pattern and classified them into many-to-many and nearest-neighbor groups. We combined contiguous, group and random job placement policies with static and adaptive routing algorithms and created six different configurations.
The goal is to examine application interference on fat-tree systems by holistically investigating the joint effects of application communication characteristics, job placement policy, and network routing mechanism. We have analyzed application communication performance under a variety of combinations of job placement policies and network routing mechanisms. Our study focuses on two types of applications, one dominant with nearest-neighbor communication and the other dominant with many-to-many communication. Our in-depth analysis results in several key observations. In particular, we find that contiguous job placement with static routing is preferred for the applications dominant with nearest-neighbor 


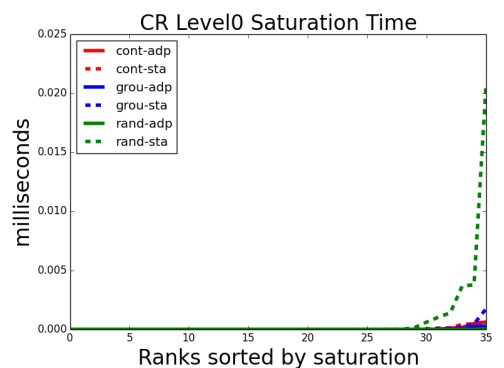

(a) Level L0

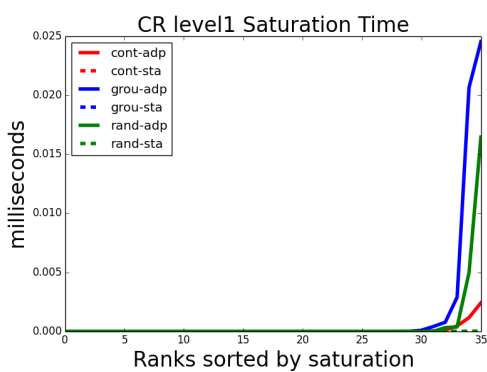

(b) Level L1

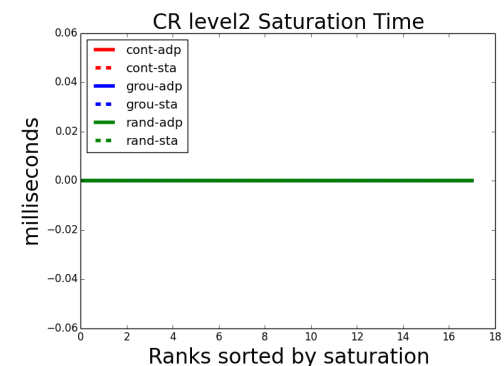

(c) Level L2

Figure 8: Saturation Time for CrystalRouter Application.

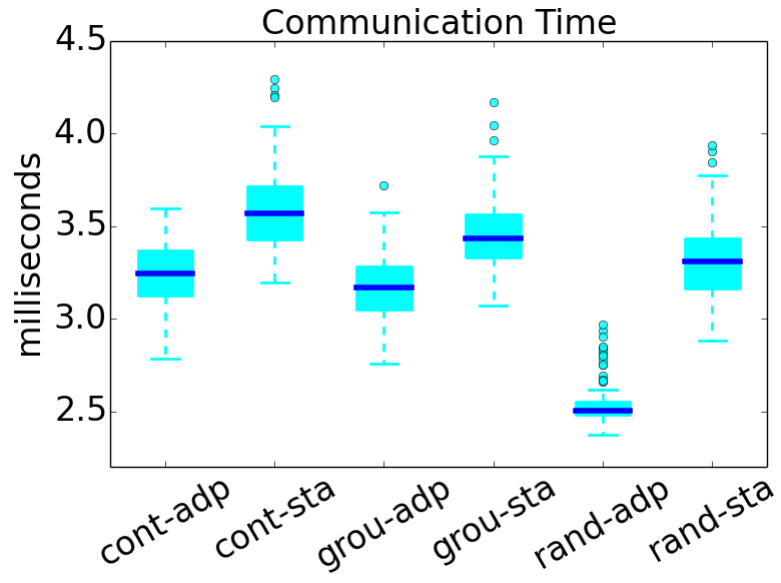

(a) Communication Time for MultiGrid Application

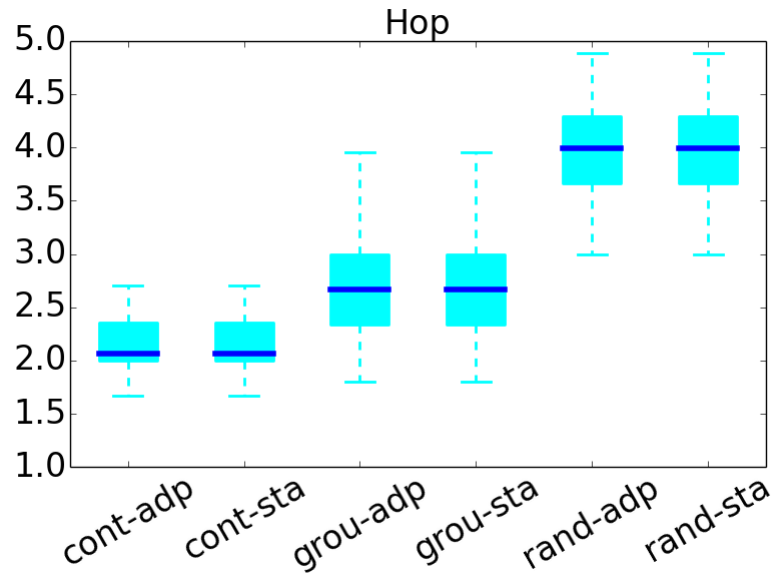

(b) Hops for MultiGrid Application.

Figure 9: Communication Time and Hops for MultiGrid Application.

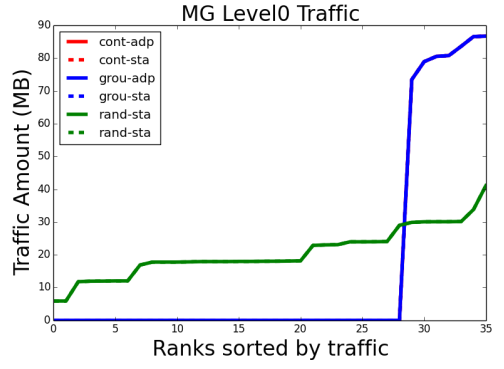

(a) Level L0

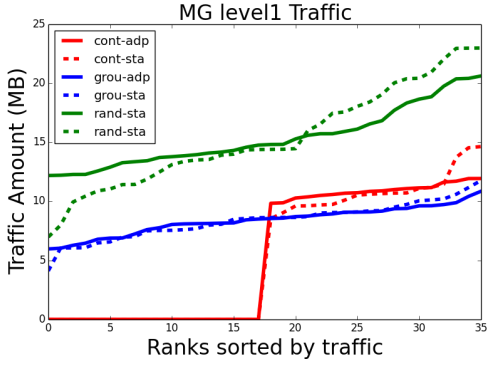

(b) Level L1

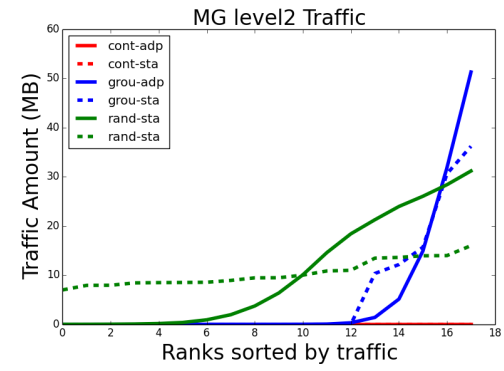

(c) Level L2

Figure 10: Traffic Amount for MultiGrid Application.

communication, whereas random job placement with adaptive routing is preferred for the applications dominant with many-to-many communication.

While this analysis provides useful insights into application interference on fat-tree systems, there are many avenues for taking this work further. A natural direction to extend our current work is to deploy and test the proposed group placement on production systems under various workloads. Another interesting avenue is to investigate workload interference among multiple applications on fat-tree systems. 


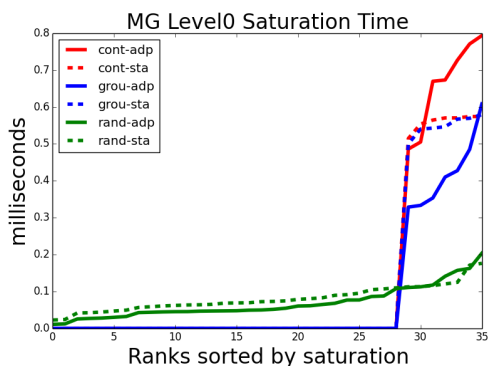

(a) Level Lo

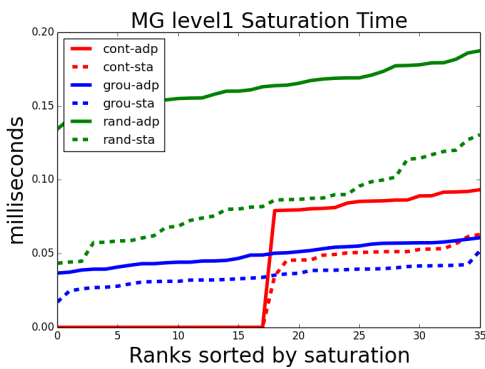

(b) Level L1

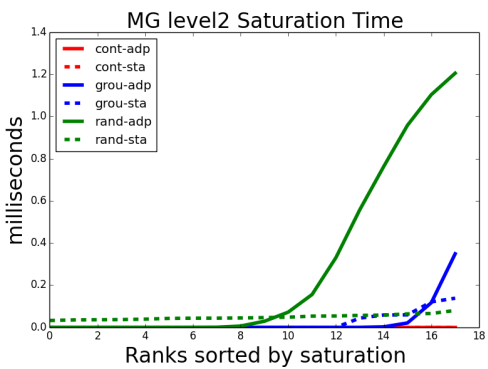

(c) Level L2

Figure 11: Saturation Time for MultiGrid Application.

\section{ACKNOWLEDGMENTS}

The authors would like to thank Misbah Mubarak, Nikhil Jain and Noah Wolfe for providing us technical support of CODES. The work is supported in part by US National Science Foundation grants CNS1717763 and CCF-1422009.

\section{REFERENCES}

[1] Mohammad Al-Fares, Alexander Loukissas, and Amin Vahdat. 2008. A scalable, commodity data center network architecture. In ACM SIGCOMM Computer Communication Review, Vol. 38. ACM, 63-74.

[2] J Bell, A Almgren, V Beckner, M Day, M Lijewski, A Nonaka, and W Zhang. 2012 BoxLib userÃćÂĂÂŹs guide. github. com/BoxLib-Codes/BoxLib (2012).

[3] Texas Advanced Computing Center. accessed Nov. 5, 2017. Stampede User Guide. https://portal.tacc.utexas.edu/user-guides/stampede

[4] Jason Cope, Ning Liu, Sam Lang, Phil Carns, Chris Carothers, and Robert Ross 2011. Codes: Enabling co-design of multilayer exascale storage architectures. In Proceedings of the Workshop on Emerging Supercomputing Technologies, Vol. 2011.

[5] Christina Delimitrou and Christos Kozyrakis. 2013. ibench: Quantifying interference for datacenter applications. In Workload Characterization (IISWC), 2013 IEEE International Symposium on. IEEE, 23-33.

[6] Jack Dongarra. 2013. Visit to the National University for Defense Technology Changsha. Oak Ridge National Laboratory.

[7] National Center for Atmospheric Research. accessed Nov. 5, 2017. YellowStone Supercomputer. https://www2.cisl.ucar.edu/resources/computational-systems/ yellowstone

[8] Ana Jokanovic, German Rodriguez, Jose Carlos Sancho, and Jesus Labarta. 2010 Impact of inter-application contention in current and future HPC systems. In High Performance Interconnects (HOTI), 2010 IEEE 18th Annual Symposium on. IEEE, 15-24.

[9] Ana Jokanovic, Jose Carlos Sancho, German Rodriguez, Alejandro Lucero, Cyriel Minkenberg, and Jesus Labarta. 2015. Quiet neighborhoods: Key to protect job performance predictability. In Parallel and Distributed Processing Symposium (IPDPS), 2015 IEEE International. IEEE, 449-459.

[10] Ana Jokanovic, Jose Carlos Sancho, German Rodriguez, Cyriel Minkenberg Ramon Beivide, and Jesus Labarta. 2013. On the trade-off of mixing scientific applications on capacity high-performance computing systems. IET Computers \& Digital Techniques 7, 2 (2013), 81-92.

[11] Melanie Kambadur, Tipp Moseley, Rick Hank, and Martha A Kim. 2012. Measuring interference between live datacenter applications. In Proceedings of the International Conference on High Performance Computing, Networking, Storage and Analysis. IEEE Computer Society Press, 51.

[12] Sandia National Laboratories. accessed Nov. 2, 2017. SST DUMPI trace library. https://github.com/sstsimulator/sst-dumpi

[13] Lawrence Livermore National Laboratory. accessed Nov. 5, 2017. Cab Supercompute. https://computation.llnl.gov/computers/cab

[14] Oak Ridge National Laboratory. accessed Nov. 5, 2017. Summit Supercomputer. https://www.olcf.ornl.gov/summit/

[15] Ning Liu, Christopher Carothers, Jason Cope, Philip Carns, Robert Ross, Adam Crume, and Carlos Maltzahn. 2012. Modeling a leadership-scale storage system. Parallel Processing and Applied Mathematics (2012), 10-19.

[16] Javier Navaridas, Jose A Pascual, and Jose Miguel-Alonso. 2009. Effects of job and task placement on parallel scientific applications performance. In Parallel, Distributed and Network-based Processing, 2009 17th Euromicro International Conference on. IEEE, 55-61.
[17] Sabine R Ohring, Maximilian Ibel, Sajal K Das, and Mohan J Kumar. 1995. On generalized fat trees. In Parallel Processing Symposium, 1995. Proceedings., 9th International. IEEE, 37-44.

[18] Sabine R Ohring, Maximilian Ibel, Sajal K Das, and Mohan J Kumar. 1995. On generalized fat trees. In Parallel Processing Symposium, 1995. Proceedings., 9th International. IEEE, 37-44.

[19] E. Merzari P. Fischer, A. Obabko and O. Marin. accessed Nov. 5, 2017. Nek5000: Computational fluid dynamics code. http://nek5000.mcs.anl.gov

[20] Philip C Roth, Jeremy S Meredith, and Jeffrey S Vetter. 2015. Automated Characterization of Parallel Application Communication Patterns. In Proceedings of the 24th International Symposium on High-Performance Parallel and Distributed Computing. ACM, 73-84.

[21] Jeffrey S Vetter and Frank Mueller. 2003. Communication characteristics of largescale scientific applications for contemporary cluster architectures. F. Parallel and Distrib. Comput. 63, 9 (2003), 853-865.

[22] Noah Wolfe, Christopher D Carothers, Misbah Mubarak, Robert Ross, and Philip Carns. 2016. Modeling a million-node slim fly network using parallel discreteevent simulation. In Proceedings of the 2016 annual ACM Conference on SIGSIM Principles of Advanced Discrete Simulation. ACM, 189-199.

[23] Noah Wolfe, Misbah Mubarak, Nikhil Jain, Jens Domke, Abhinav Bhatele, Christopher D Carothers, and Robert B Ross. [n. d.]. Preliminary Performance Analysis of Multi-rail Fat-tree Networks. ([n. d.]).

[24] Ulrike Meier Yang et al. 2002. BoomerAMG: a parallel algebraic multigrid solver and preconditioner. Applied Numerical Mathematics 41, 1 (2002), 155-177.

[25] Xu Yang, John Jenkins, Misbah Mubarak, Robert B Ross, and Zhiling Lan. 2016. Watch out for the bully! job interference study on dragonfly network. In Proceedings of the International Conference on High Performance Computing, Networking, Storage and Analysis, ser. SC, Vol. 16.

[26] Xu Yang, John Jenkins, Misbah Mubarak, Xin Wang, Robert B Ross, and Zhiling Lan. 2016. Study of intra-and interjob interference on torus networks. In Parallel and Distributed Systems (ICPADS), 2016 IEEE 22nd International Conference on. IEEE, 239-246.

[27] Xu Yang, Zhou Zhou, Wei Tang, Xingwu Zheng, Jia Wang, and Zhiling Lan. 2014. Balancing job performance with system performance via locality-aware scheduling on torus-connected systems. In Cluster Computing (CLUSTER), 2014 IEEE International Conference on. IEEE, 140-148.

[28] Eitan Zahavi. 2012. Fat-tree routing and node ordering providing contention free traffic for MPI global collectives. F. Parallel and Distrib. Comput. 72, 11 (2012), 1423-1432.

[29] Eitan Zahavi, Gregory Johnson, Darren J Kerbyson, and Michael Lang. 2010. Optimized InfiniBandTM fat-tree routing for shift all-to-all communication patterns. Concurrency and Computation: Practice and Experience 22, 2 (2010), 217-231.

[30] Zhou Zhou, Xu Yang, Zhiling Lan, Paul Rich, Wei Tang, Vitali Morozov, and Narayan Desai. 2014. Bandwidth-aware resource management for extreme scale systems. In Int. Conf. High Perform. Comput., Netw., Storage Anal.(SC14), poster session.

[31] Zhou Zhou, Xu Yang, Zhiling Lan, Paul Rich, Wei Tang, Vitali Morozov, and Narayan Desai. 2015. Improving batch scheduling on Blue Gene/Q by relaxing 5D torus network allocation constraints. In Parallel and Distributed Processing Symposium (IPDPS), 2015 IEEE International. IEEE, 439-448.

[32] Zhou Zhou, Xu Yang, Zhiling Lan, Paul Rich, Wei Tang, Vitali Morozov, and Narayan Desai. 2016. Improving Batch Scheduling on Blue Gene/Q by Relaxing Network Allocation Constraints. IEEE Transactions on Parallel and Distributed Systems 27, 11 (2016), 3269-3282. 